\title{
Comparative enantioseparation of chiral 4,4'-bipyridine derivatives on coated and immobilized amylose-based chiral stationary phases
}

\author{
Paola Peluso $^{\mathrm{a}, *}$, Barbara Sechi ${ }^{\mathrm{a}}$, Giancarlo Lai ${ }^{\mathrm{a}}$, Alessandro Dessì ${ }^{\mathrm{a}}$, Roberto Dallocchio ${ }^{\mathrm{a}}$, Sergio Cossu ${ }^{\mathrm{b}}$, \\ Emmanuel Aubert $^{\mathrm{c}}$, Robin Weiss ${ }^{\mathrm{d}}$, Patrick Pale ${ }^{\mathrm{d}}$, Victor Mamane ${ }^{\mathrm{d}, *}$, Bezhan Chankvetadze ${ }^{\mathrm{e}}$ \\ a Istituto di Chimica Biomolecolare ICB, CNR, Sede secondaria di Sassari, Traversa La Crucca 3, Regione Baldinca, Li Punti I-07100, Sassari, Italy \\ b Dipartimento di Scienze Molecolari e Nanosistemi DSMN, Università Ca' Foscari Venezia, Via Torino 155, Mestre Venezia I-30172, Italy \\ ${ }^{\mathrm{c}}$ Cristallographie, Résonance Magnétique et Modélisations (CRM2), UMR CNRS 7036, Université de Lorraine, Bd des Aiguillettes, Vandoeuvre-les-Nancy 54506, France \\ d Institut de Chimie de Strasbourg, UMR CNRS 7177, Equipe LASYROC, 1 rue Blaise Pascal, Strasbourg 67008, Cedex, France \\ e Institute of Physical and Analytical Chemistry, School of Exact and Natural Sciences, Tbilisi State University, Tbilisi, Georgia
}

\section{A R T I C L E I N F O}

\section{Article history:}

Received 26 February 2020

Received in revised form 13 May 2020

Accepted 1 June 2020

Available online xxx

\section{Keywords}

Bipyridines

Electrostatic potential

Enantiomer elution order

High-performance liquid chromatography

Immobilized amylose-based chiral stationary

phase

\begin{abstract}
A B S T R A C T
The chromatographic performances of four coated and immobilized amylose phenylcarbamate-based chiral columns were evaluated and compared under normal phase (NP) elution conditions by using chiral 4,4'-bipyridine derivatives as analytes. $n$-Hexane/2-propanol 90:10 and $n$-hexane/2-propanol/methanol 90:5:5 mixtures were employed as mobile phases (MPs), and the effect of adding methanol in the MP on retention and selectivity was considered. The effect of temperature on retention and selectivity was also evaluated, and overall thermodynamic parameters associated with the analyte adsorption onto the CSP surface were derived from van't Hoff plots. Interesting cases of enantiomer elution order (EEO) reversal, which are dependent on the nature of polar modifier, analyte structure, column-type, and temperature, were observed. The impact of substitution pattern and electronic properties of analytes and selectors on the separation behaviour was investigated by correlating chromatographic parameters and molecular properties determined by using density functional theory (DFT) calculations. Both coated and immobilized amylose tris(3,5-dimethylphenylcarbamate) columns allowed for the baseline enantioseparation $\left(2.0 \leq R_{\mathrm{S}} \leq 4.9\right)$ of all 4,4 '-bipyridines considered in this study. These results appear particularly useful because both enantiomers of these 4,4'-bipyridine derivatives are currently under investigation as new inhibitors of transthyretin fibrillogenesis, a biochemical phenomenon which is implicated to cause amyloid diseases.
\end{abstract}

\section{Introduction}

Versatility, robustness and the highest loading capability among all other chiral supports make amylose and cellulose phenylcarbamate-based chiral stationary phases (CSPs) the most used for HPLC enantioseparation of chiral compounds [1]. These advantageous features are due to the structural peculiarities of these chiral supports where the polysaccharide derivative is coated or immobilized on the silica surface with high density of chiral selector molecules per unit surface $[2,3]$. The polysaccharide phenylcarbamate is arranged in a high-ordered secondary structure forming grooves and ravines containing both polar and hydrophobic regions which accommodate and discriminate enantiomers through an elevated number of noncovalent interaction options (Fig. 1a) [4-6]. The supramolecular structure of polysaccharide-based selectors impacts their chiral recognition ability, and for

this

rea-

\footnotetext{
* Corresponding authors.

E-mail addresses: paola.peluso@cnr.it (P. Peluso); vmamane@unistra.fr (V. Mamane)
}

son, the preparation of the CSP is a critical step [1,3]. In this regard, several studies published over time reported differences in chiral recognition ability of coated and covalently immobilized polysaccharide-based selectors [7-12]. Indeed, some covalent immobilization technologies require the use of under-derivatized polysaccharides in order to attach the selector to the silica surface. Actually, all the immobilization conditions produce chemical and/or physical alteration of the selector $[3,12]$.

The study of properties, function, and mechanisms of chiral chromatographic systems involves expertise at the interface of analytical, physical and organic chemistry. On one hand, the enantioseparation process concerns the adsorption phenomenon underlying retention mechanism of analyte molecules which compete with solvent molecules onto the CSP surface $[13,14]$. On this basis, the overall chromatographic separation process derives from consecutive single adsorption and desorption steps occurring over the CSP surface during the advancement of the analyte along the column $[1,4]$. On the other hand, chiral analytes, selectors, and most mobile phase (MP) components are organic compounds, and the practical scope of an enantioseparation procedure is the resolution of racemic mixture of chiral organic com- 
(a)

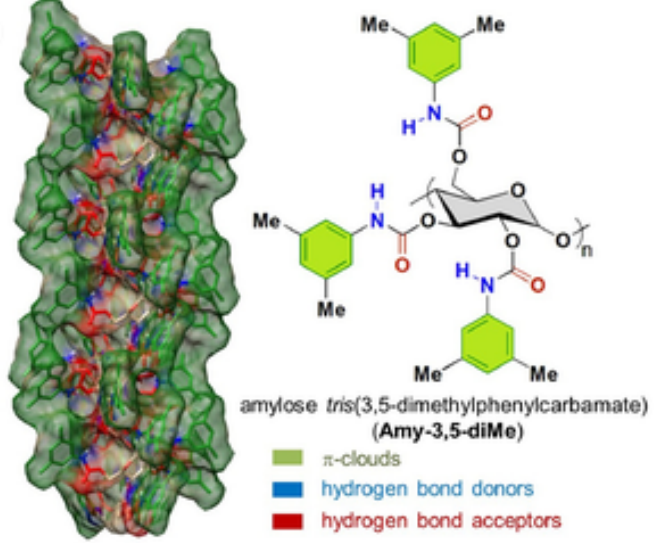

(b)

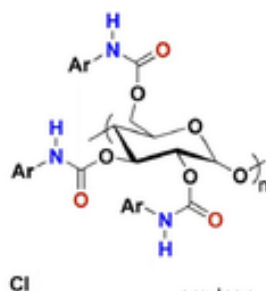

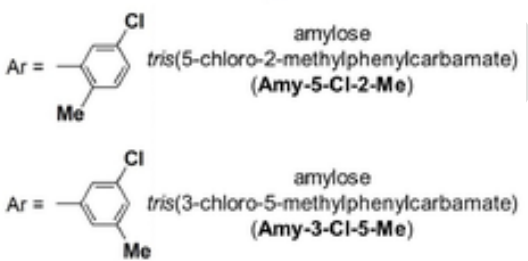

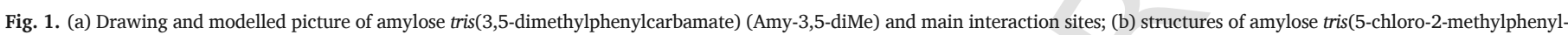
carbamate) (Amy-5-Cl-2-Me) and tris(3-chloro-5-methylphenylcarbamate) (Amy-3-Cl-5-Me). in enantiodiscrimination processes. sitions (Fig. 2b).

pounds such as pharmaceuticals, agrochemicals, food additives, fragrances, chiral pollutants, fine chemicals. As a consequence, the structural properties (shape, geometry and electronic distribution) of the three pivotal components of the chromatographic system play a key role

Given this context, we described herein the results of a comparative study on the enantioseparation of chiral 4,4'-bipyridines 1-5 and 7 (Fig. 2) by using two coated and two immobilized chiral columns containing amylose phenylcarbamate selectors (Fig. 1), under normal phase (NP) elution conditions. Compounds 1-5 and 7 are chiral by atropisomerism because of the restricted rotation around the 4,4'-biaryl bond (chiral axis) induced by the substituents located at the 3,3',5,5'-po-

The first aim of this study was to derive information on involved intermolecular contacts between chiral selector and analytes by evaluating and comparing the chromatographic outcomes as the chromatographic system is modified through subtle structural variations of analyte and selector, type of CSP, and MP composition. For this purpose, compounds 1-4 appeared to be interesting test probes to evaluate the impact of subtle structural variations on retention and selectivity. Indeed, 4,4'-bipyridines 1-4 contain a symmetric 3,3',5,5'-tetrachloro substitution pattern and an iodine atom at the 2-position as the common motif, whereas a variable substructure is located at the 2'-position (Fig. 2a), the achiral 4,4'-bipyridine 6 and the hexahalogenated 5 and 7 serving as reference compounds for comparison. (a)<smiles>[R]c1ncc(Cl)c(-c2c(Cl)cnc(I)c2Cl)c1Cl</smiles>

$1-6$

$$
\begin{aligned}
\mathrm{R}= & \mathrm{Ph}(1) \\
& 4-\text { pyridyl (2) } \\
& (p-\mathrm{OH}) \mathrm{C}_{6} \mathrm{H}_{4}(3) \\
& \mathrm{CH}_{2} \mathrm{OH}(4) \\
& \mathrm{I}(5) \\
& \mathrm{H}(6)
\end{aligned}
$$

(b)<smiles>[X]c1cnc([X])c([X])c1-c1c([X])cnc([R])c1[X]</smiles>

(M)<smiles>Clc1cnc(Cl)c(Cl)c1-c1c(Cl)cnc(Cl)c1Cl</smiles>

The four chiral columns selected for this study are characterized by the same amylose backbone which is decorated with distinctive phenylcarbamate moieties (Fig. 1), thus the impact of different structural environment on retention and selectivity could be evaluated. In addition, the impact of immobilization on retention and selectivity was evaluated by comparing the chromatographic results obtained by using both coated and immobilized chiral columns with amylose tris(3,5-dimethylphenylcarbamate) (Amy-3,5-diMe) as selector. The temperature dependence of retention and separation factors was explored and compared in order to profile possible differences between coated and immobilized CSP.

The effect of substitution pattern and electronic properties of analytes and selectors on the separation behaviour was investigated by correlating chromatographic parameters and molecular properties determined by using density functional theory (DFT) calculations [15].

Moreover, compounds 1-4 have applicative interest as they exhibited promising activity as inhibitors of transthyretin (TTR) misfolding, which is implicated in the TTR amyloidosis diseases [16], the $M$-enantiomer showing different activity with respect to the P-enantiomer (Fig. 2b). On this basis, the second aim of this investigation was to develop suitable enantioseparation methods to resolve racemic mixture of 1-4 in order to produce enantiopure forms for biological evaluation, as no asymmetric synthesis of atropisomeric 4,4'-bipyridines is available so far. In our previous studies, amylose-based CSPs were successfully used for the enantioseparation of 4,4'-bipyridine derivatives [17-20]. The chromatographic analyses were performed by using $n$-hexanic mixtures as MPs, also evaluating the effect of introducing methanol in the MP on retention and selectivity.

\section{Experimental}

\subsection{Chemicals}

Compounds 1-7 were synthesized, purified and characterized as reported $[16,20]$.

\subsection{Chromatography}

An Agilent Technologies (Waldbronn, Germany) 1100 Series HPLC system (high-pressure binary gradient system equipped with a diode-array detector operating at multiple wavelengths $(220,254,280,360 \mathrm{~nm})$, a programmable autosampler with a $20 \mu \mathrm{l}$ loop, and a thermostated column compartment) was employed for both analytical and multimilligram separations. Data acquisition and analyses were carried out with Agilent Technologies ChemStation Version B.04.03 chromatographic data software. The UV absorbance is reported as milliabsorbance units (mAU). Lux Amylose-1 (coated) and i-Amylose-1 (immobilized) (amylose tris(3,5-dimethylphenylcarbamate)

(Amy-3,5- 
diMe)), Lux Amylose-2 (amylose tris(5-chloro-2-methyl-phenylcarbamate) (coated Amy-5-Cl-2-Me), and Lux i-Amylose-3 (amylose tris(3-chloro-5-methyl-phenylcarbamate) (immobilized Amy-3-Cl-5-Me)) were used as chiral columns $(5 \mu \mathrm{m}, 250 \times 4.6 \mathrm{~mm}$ ) (Phenomenex Inc., Torrance, CA, USA). HPLC grade $n$-hexane (Hex), isopropanol (IPA), and methanol $(\mathrm{MeOH})$ were purchased from Sigma-Aldrich (Taufkirchen, Germany). The retention factor $(k)$ was determined as $k=\left(t_{\mathrm{R}}-t_{0}\right) / t_{0}$, where $t_{\mathrm{R}}$ is the retention time for the eluted enantiomer; $k_{1}$ is the retention factor of the first-eluted enantiomer. The separation factor $(\alpha)$ was calculated as $\alpha=k_{2} / k_{1}$. Analyses were performed in isocratic mode at $25^{\circ} \mathrm{C}$. The flow rate $(F R)$ was set at $0.8 \mathrm{ml} / \mathrm{min}$. Dead time $\left(t_{0}\right)$ was measured by injection of tri-tertbutylbenzene (Sigma-Aldrich) as a non-retained compound [21]: $t_{0}=3.50 \mathrm{~min}$ (coated Amy-3,5-diMe), $3.61 \mathrm{~min}$ (immobilized Amy-3,5-diMe), 3.63 min (coated Amy-5-Cl-2-Me), 3.66 min (immobilized Amy-3-Cl-5-Me). The enantiomer elution order (EEO) was determined by injecting enantiomers of known absolute configuration. Pure enantiomers of compounds 1-7 were obtained by HPLC enantioseparation and their absolute configuration was assigned on the basis of X-ray diffraction or by comparison of theoretical/experimental electronic circular dichroism spectra, as previously described $[16,20]$. The van't Hoff experiments were conducted at 5, 10, 15, 20, 25, 30, 35,40 , and $45^{\circ} \mathrm{C}$ by using a thermostat jacket equipped with a RE104 LAUDA circulating water-bath (Lauda, Königshofen, Germany) (resolution $0.1^{\circ} \mathrm{C}$; accuracy $\pm 0.4^{\circ} \mathrm{C}$; temperature control $\pm 0.02^{\circ} \mathrm{C}$ ). When the temperature was changed, the column was allowed to equilibrate for $1 \mathrm{~h}$ before injecting the samples. Thermodynamic parameters were derived from the slopes and the intercepts of the van't Hoff plots by linear regression analysis. Statgraphics Centurion XVI (Statpoint Technologies, Inc., Warrenton, VA, USA) was used for all linear regression analyses (Least-squares method).

\subsection{Computationals}

The 3D structures of compounds 1-7 and methyl 3,5-dimethylphenylcarbamate, methyl 3-chloro-5-methylcarbamate, and methyl 5-chloro-2-methylphenylcarbamate, as frameworks representing the CSP side chains, were prepared using the build function, and model kits and tools provided by Spartan' 10 Version 1.1.0 (Wavefunction Inc., Irvine, CA, USA) [22] for building and editing organic molecules. On this basis, molecular structures were generated and their refinement was performed by a MMFF procedure. Then, each structure was submitted to a conformational systematic search using MMFF, spanning all shapes accessible to the molecule without regard to energy. After the elimination of duplicates and high-energy conformers, a set of energetically accessible conformers was selected. For each conformer, geometry optimization was performed employing the DFT method with the B3LYP functional and the 6-311G* basis set, and finally the respective Boltzmann distribution was constructed. Geometry optimization and computation of electrostatic potential isosurfaces $\left(V_{S}\right)$ and related parameters ( $V_{\mathrm{S}}$ extrema, $V_{\mathrm{S}, \text { max }}$ and $V_{\mathrm{S} \text {,min }}$ values, given in $\left.\mathrm{kJ} / \mathrm{mol}\right)$ were performed by using Gaussian 09 (DFT, B3LYP, 6-311G*) (Wallingford, CT 06492, USA) [23]. Search for the exact location of such $V_{S \text {, max }}$ and $V_{\mathrm{S} \text {,min }}$ was made through the Multiwfn code [24] and through its module enabling quantitative analyses of molecular surfaces (isovalue 0.002 ) [25]. The $V_{S}$ maps were graphically generated through the graphical interface of Spartan ' 10 and, by convention, colors toward red depict negative potential, while colors toward blue depict positive potential, and colors in between (orange, yellow, green) depict intermediate values of potential.

\section{Results and discussion}

\subsection{Analyte structures}

In compounds 1-5 and 7, as a consequence of the atropisomeric motif, the substituents on each heteroaromatic ring present an orthogonal orientation (Fig. 3a), therefore the anisotropic properties of the chiral 4,4'-bipyridines strongly depend on the stereoelectronic features of these substituents. Indeed, in our previous studies, the enantioselectivity of 4,4'-bipyridine derivatives on polysaccharide-based CSPs proved to be strongly influenced by the nature of distinctive groups $\mathrm{R}$ and halogens located in the region around the chiral axis [17-20,26,27]. On the contrary, the direct contribution to retention and selectivity of the 4,4'-bipyridine core was shown to be low due to the weakness of the pyridine nitrogens as hydrogen bond (HB) acceptors. However, the electron-withdrawing heteroaromatic substructure polarizes bound atoms and groups, thus contributing to their capability to exert noncovalent interactions underlying chiral recognition in HPLC environment. On this basis, with the aim to estimate the extent of the abovementioned structural effects, we computed the electrostatic potential values $(V$ ) for compounds 1-7. The calculated values were then mapped on electron density isosurfaces $\left(V_{\mathrm{S}}\right)$ in order to inspect analyte shapes which result from respective geometry and electronic distribution. In Fig. 3b the calculated $V_{S}$ of compounds 2,3 , and 4 are depicted as representative cases (see Supplementary data, Tables S1-S3 for complete $V_{S}$ analysis of compounds 1-7). This kind of analysis provides information on specific regions of the molecules, such as lone pairs and $\pi$-clouds, that could participate in noncovalent interactions. In particular, regions with high (nucleophile) and low (electrophile) electron density can be identified by the negative minima $\left(V_{\mathrm{S}, \min }\right)$ and positive maxima $\left(V_{\mathrm{S}, \max }\right)$ values of $V_{\mathrm{S}}$, respectively. Recently, $V$ analysis has been fruitfully used to gain insights on selector/analyte contacts by evaluating the electron density on molecular regions involved in the noncovalent interactions [28-30].

All compounds 1-7 contain a common 3,3',5,5'-tetrachlorinated motif which represents a symmetric hydrophobic region surrounding the chiral axis (Fig. 3). This feature was expected to be detrimental for the enantioseparation under standard NP elution conditions. In addition, another hydrophobic region is present at the 2-position where iodine (1-6) or chlorine (7) atoms are located. These halogens can also act as halogen bond (XB) donors interacting through their electrophilic (a)

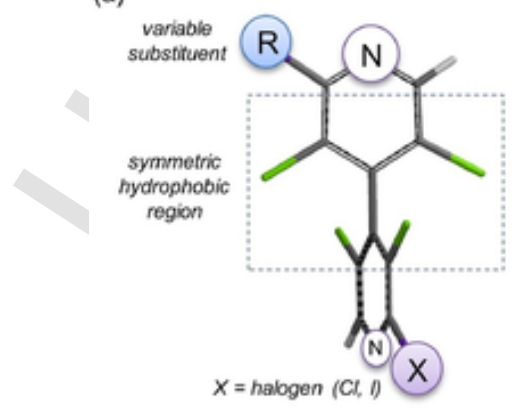

(b)

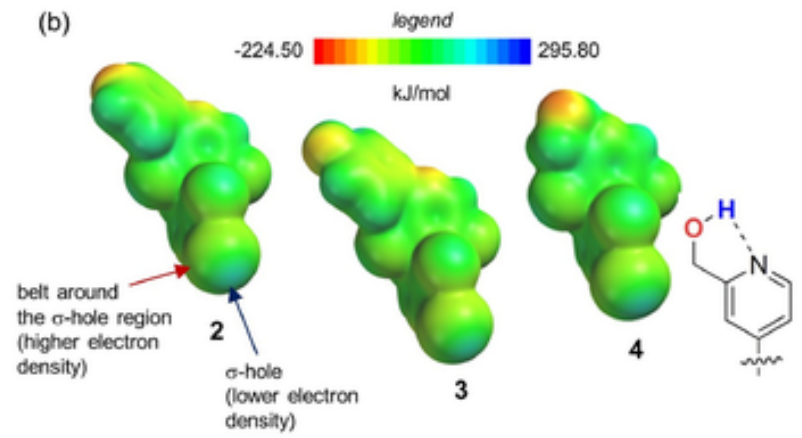

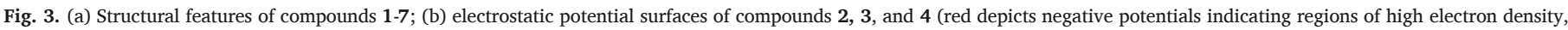
while blue depicts positive potential indicating regions of low electron density, and colours in between depict intermediate values of electrostatic potential). 
$\sigma$-holes $\left(130.1 \mathrm{~kJ} / \mathrm{mol} \leq V_{\mathrm{S}, \max }(\mathrm{I}) \leq 141.6 \mathrm{~kJ} / \mathrm{mol} ; V_{\mathrm{S}, \max }(\mathrm{Cl})=75.0\right.$ $\mathrm{kJ} / \mathrm{mol}$ ) with the nucleophilic regions of the CSPs (Fig. 3b). XB is a noncovalent interaction occurring between a Lewis base (XB acceptor) and an electropositive region (the so-called $\sigma$-hole) located on bound halogens (XB donors) [31]. In our previous studies, we demonstrated by chromatographic and computational analyses that the carbonyl oxygens of Amy-3,5-diMe and cellulose-3,5-diMe are able as Lewis bases to form XBs with the electrophilic $\sigma$-hole regions of halogen substituents (XB donors) bound to the 4,4 '-bipyridine rings $[6,20,26]$. Moreover, in principle, halogens can also act as $\mathrm{HB}$ acceptors through the electron density located on the belt around the $\sigma$-hole region (Fig. 3b) [6]. Nevertheless, in compounds 1-7, other more negative regions are present with better capability as $\mathrm{HB}$ acceptors. More important are the distinctive substituents located at 2'-position of these 4,4'-bipyridine derivatives: a phenyl ring in 1, a 4-pyridyl ring in $\mathbf{2}$ and a 4-hydroxyphenyl group in 3. In addition, in compounds 2 (2'-(4-pyridyl)) and 3 (2'-(4-hydroxyphenyl), HB sites are located on the aromatic substituents in 2'-position, a nitrogen as $\mathrm{HB}$ acceptor $\left(V_{\mathrm{S}, \min }=-173.0 \mathrm{~kJ} / \mathrm{mol}\right)$ and a hydroxyl group as $\mathrm{HB}$ acceptor/donor $\left(V_{\mathrm{S}, \min }=-110.6 \mathrm{~kJ} / \mathrm{mol} ; V_{\mathrm{S}, \max }=296.1\right.$ $\mathrm{kJ} / \mathrm{mol}$ ), respectively. The presence of $\mathrm{HB}$ sites in the 2'-moieties, located far from the chiral axis, is expected to be a second detrimental factor for enantiodiscrimination, whereas it could contribute to increase retention on the CSPs. Compound 4 contains a 2'-hydroxymethyl group as distinctive substituent which could act as HB acceptor/donor. Nevertheless, it is characterized by an intramolecular HB involving the hydrogen of the 2'-hydroxymethyl moiety and the 1'-nitrogen on the pyridyl' ring (Fig. 3b), thus limiting the availability of the hydroxyl group as $\mathrm{HB}$ donor.

Due to the atropisomer topology of 1-5 and 7, it was expected that the enantiodiscrimination degree should be related to the strength of noncovalent interactions involving both 2 - and 2'-positions.

\subsection{Amylose-based selectors}

Chiral columns based on coated and immobilized Amy-3,5-diMe (Fig. 1a), coated Amy-5-Cl-2-Me, and immobilized Amy-3-Cl-5-Me (Fig. 1b) were selected for this study in order to evaluate the impact of aryl chlorination and covalent immobilization on the enantioseparation performances of these CSPs toward rac-1-4, using compounds 5-7

Table 1

$V_{\mathrm{S}, \max }$ and $V_{\mathrm{S} \text {,min }}$ values $[\mathrm{kJ} / \mathrm{mol}]$ and related surface geometrical parameters for the amylose-based CSP side chains.

\begin{tabular}{llll}
\hline Descriptor & Amy-3,5-diMe & Amy-5-Cl-2-Me & Amy-3-Cl-5-Me \\
\hline$V_{\mathrm{S}, \min }(\mathrm{C}=\mathrm{O})$ & -165.6 & -154.9 & -148.2 \\
$V_{\mathrm{S}, \max }(\mathrm{N}-\mathrm{H})$ & 217.3 & 219.7 & 238.0 \\
$V_{\mathrm{S}, \min }(\mathrm{Ar})$ & -79.5 & -55.1 & -50.3 \\
$V_{\mathrm{S}}$ area $\left(\AA^{2}\right)$ & 216.0 & 211.4 & 214.0 \\
$V_{\mathrm{S}}$ volume $\left(\AA^{3}\right)$ & 200.0 & 197.7 & 198.4 \\
\hline
\end{tabular}

as terms of comparison. All columns contain selectors based on the same amylose backbone which is derivatized with distinctive side chains determining the stereoelectronic properties of each selector [1,32,33].

The effect of introducing chlorine is to modify the electron density distribution on the side chain moiety, thus the electron density on both the $\mathrm{C}=\mathrm{O}$ and phenyl ring decreases ( $\pi$-acidity increases), and the acidity of the $\mathrm{N}-\mathrm{H}$ increases. This trend has been confirmed by calculating $V_{\mathrm{S} \text {,max }}$ and $V_{\mathrm{S}, \min }$ values on pivotal regions of the side chains of Amy-3,5-diMe, Amy-5-Cl-2-Me, and Amy-3-Cl-5-Me (Table 1). Moreover, it was observed through IR analyses that the introduction of chlorine increases the fraction of free $\mathrm{N}-\mathrm{H}$ groups [33]. In the meantime, the fraction of $\mathrm{N}-\mathrm{H}$ involved in intramolecular $\mathrm{HBs}$, contributing to maintain the high-ordered structure of the CSP, decreases. This could produce for the chlorinated CSPs a wider cavity available for the enantiomers with respect to the dimethylated selector, the enantioseparation resulting from the balance of carbamate polarity and intramolecular HB ability [33]. Coherently, for both chlorinated side chains of Amy-3-Cl-5-Me and Amy-5-Cl-2-Me, we calculated lower values of molecular area and volume with respect to the 3,5-dimethylated side chain (Table 1).

\subsection{Chromatographic screening with n-hexane/2-propanol mixture as} mobile phase

In the first step of this study, the enantioseparability of 4,4'-bipyridines 1-5, 7 and retention of the achiral 6, were tested on coated and immobilized Amy-3,5-diMe, coated Amy-5-Cl-2-Me and immobilized Amy-3-Cl-5-Me columns by using Hex/IPA 90:10 as MP. A comparison between the behaviours of the four columns is reported in Table 2.

Concerning compounds 1-4, moderate enantioseparation was obtained for 1 ( 2 '-R = phenyl) on both Amy-3,5-diMe columns, and for $\mathbf{3}$ ( 2 '- $\mathrm{R}=4$-hydroxyphenyl) only on the coated one, whereas the selectivity values for $\mathbf{2}$ ( 2 '- $\mathrm{R}=4$-pyridyl) and 4 ( 2 '- $\mathrm{R}=$ hydroxymethyl) were unsatisfactory on all columns. Retention and selectivity were shown to be lower on the immobilized Amy-3,5-diMe with respect to the coated column, with the exception of compounds $\mathbf{1}$ and $\mathbf{2}$ which were better enantioseparated on the immobilized Amy-3,5-diMe. As general trend, compounds $\mathbf{1}, \mathbf{5}, \mathbf{6}$, and $\mathbf{7}$ showed retention values lower than compounds 2, 3 and 4, this fact proving the contribution of HB sites to retention. On the contrary, selectivity exhibited a specific trend on each column, compound 5 ( 2 '- $\mathrm{R}=$ iodine) being enantioseparated with the highest selectivity values on all columns. Compound 5 presents two positive $\sigma$-holes centred on the 2- and 2'-iodine substituents $\left(V_{\mathrm{S}, \max }=140.9 \mathrm{~kJ} / \mathrm{mol}\right)$ which are involved in XB interactions underlying enantiodiscrimination [20,26]. In most cases, higher retention and enantioselectivity were observed on both Amy-3,5-diMe columns with respect to the chlorinated columns, with the exception of compounds 2 and 4 . Indeed, for compound 4 the highest selectivity value was obtained on coated Amy-5-Cl-2-Me ( $\alpha=1.09$ ), and compound 2 showed higher retention on both chlorinated columns $\left(k_{1}=4.52\right.$

Table 2

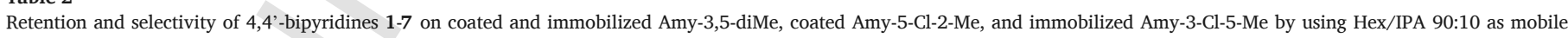
phase, $F R 0.8 \mathrm{ml} / \mathrm{min}, \mathrm{T}=25^{\circ} \mathrm{C}$ ) (the absolute configuration of eluted enantiomers is reported in brackets).

\begin{tabular}{|c|c|c|c|c|c|c|c|c|c|c|c|c|}
\hline \multirow[b]{2}{*}{ Bipy } & \multicolumn{2}{|c|}{ coated Amy-3,5-diMe } & \multirow[b]{2}{*}{$\alpha$} & \multicolumn{3}{|c|}{ immobilized Amy-3,5-diMe } & \multicolumn{3}{|c|}{ coated Amy-5-Cl-2-Me } & \multicolumn{3}{|c|}{ immobilized Amy-3-Cl-5-Me } \\
\hline & $k_{1}$ & $k_{2}$ & & $k_{1}$ & $k_{2}$ & $\alpha$ & $k_{1}$ & $k_{2}$ & $\alpha$ & $k_{1}$ & $k_{2}$ & $\alpha$ \\
\hline 1 & $1.08(P)$ & $1.32(M)$ & 1.22 & $0.87(P)$ & $1.10(M)$ & 1.26 & $0.85(P)$ & $0.92(M)$ & 1.08 & $0.76(P)$ & $0.82(M)$ & 1.08 \\
\hline 2 & $3.00(M)$ & $3.12(P)$ & 1.04 & $2.57(P)$ & $2.80(M)$ & 1.09 & $4.52(P)$ & $4.77(M)$ & 1.05 & 3.51 & 3.51 & 1.00 \\
\hline 3 & $6.50(M)$ & $8.18(P)$ & 1.26 & 4.93 & 4.93 & 1.00 & $3.44(P)$ & $3.73(M)$ & 1.09 & $2.61(P)$ & $2.79(M)$ & 1.07 \\
\hline 4 & $3.18(P)$ & $3.29(M)$ & 1.03 & $2.38(M)$ & $2.45(P)$ & 1.03 & $2.87(P)$ & $3.12(M)$ & 1.09 & 2.25 & 2.25 & 1.00 \\
\hline 5 & $1.00(M)$ & $1.63(P)$ & 1.63 & $0.76(M)$ & $1.16(P)$ & 1.53 & $0.91(P)$ & $1.00(M)$ & 1.11 & $0.64(M)$ & $0.73(P)$ & 1.14 \\
\hline 6 & 1.20 & - & - & 0.95 & - & - & 0.88 & - & - & 0.75 & - & - \\
\hline 7 & $0.46(M)$ & $0.65(P)$ & 1.41 & $0.42(M)$ & $0.58(P)$ & 1.38 & $0.57(P)$ & $0.61(M)$ & 1.06 & $0.39(M)$ & $0.42(P)$ & 1.08 \\
\hline
\end{tabular}


on coated Amy-5-Cl-2-Me and $k_{1}=3.51$ on immobilized Amy-3-Cl-5-Me) with respect to the Amy-3,5-diMe columns $\left(k_{1}=3.00\right.$; 2.57). It is likely that this trend is related to the presence of a distinctive $\mathrm{HB}$ acceptor on both 2 and 4 interacting with the $\mathrm{N}-\mathrm{H}$ of the CSP. The strength of this $\mathrm{HB}$ is definitely enhanced on the chlorinated selectors which contain better HB donor regions (Amy-5-Cl-2-Me: $V_{\mathrm{S}, \max }=219.7$ $\mathrm{kJ} / \mathrm{mol}$; Amy-3-Cl-5-Me: $238.0 \mathrm{~kJ} / \mathrm{mol}$ ) with respect to the 3,5-dimethylated selector (Amy-3,5-diMe: $V_{\mathrm{S} \text {, } \max }=217.3 \mathrm{~kJ} / \mathrm{mol}$ ). Coherently, on both Amy-3,5-diMe columns the highest retention value was observed for compound 3 which contains a positive region centred on the hydroxyl substituent, as a HB donor $\left(V_{S, \max }=296.1 \mathrm{~kJ} / \mathrm{mol}\right)$, interacting with the carbonyl oxygen of the CSP. On Amy-3,5-diMe, it is also interesting to note the correlation between retention and $V_{\mathrm{S} \text {, min }}$ calculated for nitrogen and oxygen in the 2'-substituent of 2 and 4, respectively (2: $k_{1}=3.00, V_{\mathrm{S}, \min }=-173.0 \mathrm{~kJ} / \mathrm{mol} ; 4: k_{1}=3.18, V_{\mathrm{S}, \min }=-173.8 \mathrm{~kJ} /$ $\mathrm{mol})$.

Several cases of enantiomer elution order (EEO) reversal, which are dependent on analyte structure and column-type, were observed. The EEO remained $P-M$ for all compounds only in the case of coated Amy-5-Cl-2-Me, whereas only compound 1 (2'-R = phenyl) showed $P-M$ as the elution sequence on all columns. Compounds $5(2$ ' $-\mathrm{R}=$ iodine $)$ and 7 (hexachlorinated) showed EEO $M-P$ on all columns, with the exception of coated Amy-5-Cl-2-Me. For compounds 2 (2'-R = 4-pyridyl) and 4 ( 2 '-R $=$ hydroxymethyl) EEO reversal dependent on the nature of the 2'-substituent was observed on both Amy-3,5-diMe columns. Interestingly, EEO reversal was also observed between coated and immobilized Amy-3,5-diMe for compounds 2 and 4, whereas compound 3 (2'- $\mathrm{R}=4$-hydroxyphenyl) was enantioseparated on the coated column but not on its immobilized version. On the basis of these first results, it emerged that low/moderate enantioselectivity could be achieved for compounds 1-4 with the binary mixture Hex/IPA. Moreover, immobilization of Amy-3,5-diMe selector seemed to impact the carbamate region, which is involved in $\mathrm{HB}$ interactions with the analytes, more than the substituted aromatic rings.

\subsection{Effect of introducing methanol in the mobile phase on the separation of enantiomers}

The ternary mixture Hex/IPA/MeOH 90:5:5 was used in order to optimize the analytical method, and the results are summarized in Table 3. It was expected that the MeOH-containing MP could have a beneficial effect on the enantioseparation due to the hydrophobic nature of the tetrachlorinated region around the 4,4'-chiral axis, because hydrophobic interactions are favoured in $\mathrm{MeOH}$ compared to IPA $[17,18,34]$. Moreover, $\mathrm{MeOH}$ could better favour the fit of the analytes into the chiral groove than IPA.

Indeed, the enantioseparation of compounds 1-4 were improved using $\mathrm{MeOH}$-containing MP with the Amy-3,5-diMe columns and the immobilized Amy-3-Cl-5-Me. In particular, compound 3 was enantioseparated on the immobilized Amy-3,5-diMe, which was unable to resolve this compound with the mixture Hex/IPA 90:10 (Supplementary data, Fig. S1). For compounds 1-3, because HBs were weakened due to the presence of $\mathrm{MeOH}$ in the MP, chiral recognition appeared controlled by $\pi-\pi$ interactions involving the 2'-aryl substituent. This hypothesis is confirmed by the fact that all compounds 1-3 showed the same EEO $(P-M)$. Moreover, lower selectivity was observed for compound 2 (2'-R = 4-pyridyl) with respect to $\mathbf{1}(2$ '- $\mathrm{R}=$ phenyl) and $\mathbf{3}$ ( 2 '-R $=4$-hydroxyphenyl) on coated Amy-3,5-diMe and immobilized Amy-3-Cl-5-Me, which could be correlated to the positive electrostatic potential calculated for the 4-pyridyl 2'-substituent $\left(V_{\mathrm{S}, \min }=5.12 ; 7.55\right.$ $\mathrm{kJ} / \mathrm{mol}$ ) ( $\pi$-acid aromatic ring), whereas negative electrostatic potential values were found for $\mathbf{1}$ and 3, ranging from -28.8 to $-40.1 \mathrm{~kJ} / \mathrm{mol}$ ( $\pi$-basic aromatic ring). In contrast, the effect of introducing $\mathrm{MeOH}$ in the MP was detrimental for the enantioseparation of compounds 1-4 on Amy-5-Cl-2-Me, and for 4 (2'-R = hydroxymethyl) on the immobilized Amy-3,5-diMe column.

More specifically, it is interesting to note that the use of $\mathrm{MeOH}$ was unfavourable for retention and selectivity of the halogenated compounds 5 (2'-R = iodine) and 7 (hexachlorinated), and for retention of $6\left(2^{\prime}-\mathrm{R}=\mathrm{H}\right)$. This negative effect on retention and selectivity was expected due to the interaction of $\mathrm{MeOH}$ with the carbonyl oxygens of the polysaccharide selectors, thus competing with the XBs which underlie interaction between halogenated 4,4'-bipyridines and these carbonyl oxygens [6].

The enantioseparation results obtained with the ternary mixture $\mathrm{Hex} / \mathrm{IPA} / \mathrm{MeOH}$ confirmed the hypothesis that immobilization of Amy-3,5-diMe selector affects in particular the ability of the carbamate moiety to exert HBs. Indeed, with the MeOH-containing MP the two Amy-3,5-diMe columns tend to behave in a comparable manner, the introduction of $\mathrm{MeOH}$ being detrimental for HBs occurring between analyte and selector.

\subsection{Effect of temperature on the enantioseparation with coated and covalently immobilized chiral selectors}

The nature of analyte/CSP association can be explored on the basis of thermodynamic considerations. Theory of adsorption phenomena in chromatography, and methods for profiling temperature dependence of retention and selectivity and calculating thermodynamic quantities associated with the adsorption of analytes on the CSP surface have been the matter of several papers [13,14,35-40].

The van't Hoff equation (Eq. (1)) allows for determining the macroscopic thermodynamic quantities governing enantiomer adsorption and enantioseparation $[11,41,42]$. This equation describes the dependence between retention factor $\mathrm{k}$ and the absolute temperature $\mathrm{T}$ :

$\ln k=-\Delta H^{\circ} / R \mathrm{~T}+\Delta S^{\circ} / R+\ln \Phi$

where $R$ is the gas constant $\left(1.987 \mathrm{cal} \bullet \mathrm{K}^{-1} \mathrm{~mol}^{-1}\right)$ and $\Phi$ is the phase ratio. $\Delta H^{\circ}$ and $\Delta S^{\circ}$ represent the differences in the enthalpy and entropy, respectively, when one enantiomer is adsorbed onto the CSP surface. Assuming that the plots of $\ln k$ against $1 / \mathrm{T}$ is linear in the temperature range of a study, the correlative thermodynamic parame-

Table 3

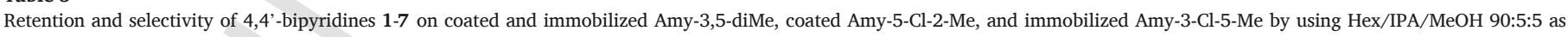
mobile phase, $F R 0.8 \mathrm{ml} / \mathrm{min}, \mathrm{T}=25^{\circ} \mathrm{C}$ (the absolute configuration of eluted enantiomers is reported in brackets).

\begin{tabular}{|c|c|c|c|c|c|c|c|c|c|c|c|c|}
\hline \multicolumn{4}{|c|}{ coated Amy-3,5-diMe } & \multicolumn{3}{|c|}{ immobilized Amy-3,5-diMe } & \multicolumn{3}{|c|}{ coated Amy-5-Cl-2-Me } & \multicolumn{3}{|c|}{ immobilized Amy-3-Cl-5-Me } \\
\hline Bipy & $k_{1}$ & $k_{2}$ & $\alpha$ & $k_{1}$ & $k_{2}$ & $\alpha$ & $k_{1}$ & $k_{2}$ & $\alpha$ & $k_{1}$ & $k_{2}$ & $\alpha$ \\
\hline 1 & $0.61(P)$ & $0.82(M)$ & 1.34 & $0.72(P)$ & $0.94(M)$ & 1.31 & 0.65 & 0.65 & 1.00 & $0.54(P)$ & $0.64(M)$ & 1.18 \\
\hline 2 & $1.96(P)$ & $2.54(M)$ & 1.29 & $2.31(P)$ & $2.79(M)$ & 1.21 & 3.10 & 3.10 & 1.00 & $2.82(P)$ & $3.08(M)$ & 1.09 \\
\hline 3 & $2.26(P)$ & $3.24(M)$ & 1.43 & $2.50(P)$ & $3.30(M)$ & 1.32 & 1.80 & 1.80 & 1.00 & $1.45(P)$ & $1.87(M)$ & 1.29 \\
\hline 4 & $1.80(M)$ & $1.93(P)$ & 1.07 & 1.84 & 1.84 & 1.00 & 1.88 & 1.88 & 1.00 & $1.64(P)$ & $1.71(M)$ & 1.04 \\
\hline 5 & $0.63(M)$ & $1.00(P)$ & 1.58 & $0.69(M)$ & $0.78(P)$ & 1.13 & $0.68(P)$ & $0.73(M)$ & 1.07 & 0.55 & 0.55 & 1.00 \\
\hline 6 & 0.77 & - & - & 0.68 & - & - & 0.64 & - & - & 0.59 & - & - \\
\hline 7 & $0.34(M)$ & $0.46(P)$ & 1.38 & $0.40(M)$ & $0.44(P)$ & 1.10 & 0.39 & 0.39 & 1.00 & 0.31 & 0.31 & 1.00 \\
\hline
\end{tabular}


ters, which are temperature-independent, can be derived from the slope $\left(\Delta H^{\circ}=-\right.$ slope $\left.\times R\right)$ and the intercept $\left(\Delta S^{*}=\right.$ intercept $\times R$, where $\Delta S^{*}$ is used to substitute the expression $\Delta S^{\circ} / R+\ln \Phi$ ) of the straight lines. The free energy associated with the adsorption of an enantiomer onto the CSP surface is given by the Gibbs-Helmholtz Eq. (2):

$\Delta G^{\circ}=\Delta H^{\circ}--\mathrm{T} \Delta S^{\circ}$

$\Delta \Delta G^{\circ}, \Delta \Delta H^{\circ}$ and $\Delta \Delta S^{\circ}$ represent the difference between the free energy of adsorption of the two enantiomers and its enthalpic and entropic terms, respectively (Eq. (3)):

$\Delta \Delta G^{\circ}=\Delta \Delta H^{\circ}--\mathrm{T} \Delta \Delta S^{\circ}$

On the basis of the van't Hoff Eq. (1), $\Delta \Delta H^{\circ}$ and $\Delta \Delta S^{\circ}$ can be derived from Eq. (4):

$\ln a=-\Delta \Delta H^{\circ} / R \mathrm{~T}+\Delta \Delta^{\circ} S / R$

The knowledge of $\Delta \Delta H^{\circ}$ and $\Delta \Delta S^{\circ}$ allows for calculating the isoenantioselective temperature $\left(\mathrm{T}_{I S O}\right)$ :

$\mathrm{T}_{I S O}=\Delta \Delta H^{\circ} / \Delta \Delta S^{\circ}$

At this temperature, the enthalpic and the entropic terms of Eq. (3) compensate each other, the free energy term is zero and the enantiomers co-elute. In general, enantioseparations at lower temperatures than $\mathrm{T}_{I S O}$ are enthalpy-driven $\left(\left|\Delta H^{\circ}\right|>\left|\mathrm{T} \Delta S^{\circ}\right|\right)$, whereas at higher temperatures enantioseparations are entropy-driven $\left(\left|\Delta H^{\circ}\right|<\left|\mathrm{T} \Delta S^{\circ}\right|\right)$. By changing the temperature between the two regions, an EEO reversal occurs.

On one hand, the thermodynamic quantities derived by classical van't Hoff Eq. (1) are macroscopic entities which do not account for surface heterogeneity of the CSPs, and this type of analysis does not allow for determining individually achiral and chiral features of enantioseparation and their actual ratio in the discrimination process $[14,36,40]$. Indeed, in principle, CSPs contain two types of adsorption sites, non-enantioselective sites (type I) and enantioselective sites (type II) $[37,43]$. In this regard, several methodologies have been described for determining adsorption isotherms accounting for site-selective thermodynamics [14,38]. Therefore, experiments and data interpretation in this field have to be carefully managed and performed in order to avoid misleading conclusions $[39,40]$. On the other hand, even if the molar quantities determined on the basis of Eq. (1) are, as a matter of fact, composite values representing different adsorption types, a huge number of studies has successfully applied this method to profile and compare temperature dependence of different chiral supports, also achieving meaningful practical information on the overall adsorption process $[4,11,41,42,44]$. In this regard, it is interesting to note that thermodynamic parameters are depending on analyte, CSP and MP, therefore useful information can emerge by comparison of thermodynamic data of analogue analyte/CSP pairs as subtle variations of the chromatographic system (analyte, CSP, MP) occur. Moreover, temperature impacts the chromatographic behaviour of analyte in HPLC enantioseparation, therefore it should be considered as a variable to optimize separations $[11,44]$.

In this perspective, with the aim to compare the thermodynamic profiles of coated and immobilized CSPs as derived from van't Hoff analysis, retention and selectivity of compounds $2-4$, on coated and immobilized Amy-3,5-diMe, and the immobilized Amy-3-Cl-5-Me, were determined at different temperatures from 5 to $45^{\circ} \mathrm{C}$ in $5^{\circ} \mathrm{C}$ increments (Supplementary data, Tables S6-S10). The following mixtures were used as MPs: Hex/IPA 90:10 and Hex/IPA/MeOH 90:5:5 for coated Amy-3,5-diMe and immobilized Amy-3,5-diMe, and Hex/IPA 90:10 for immobilized Amy-3-Cl-5-Me. The thermodynamic quantities derived from van't Hoff plots (Figs. 4-6, S2 and S5) are reported in Table S11-S15 (Supplementary data). From the analysis performed on both coated and immobilized Amy-3,5-diMe with Hex/IPA 90:10 as MP, interesting remarks emerged:
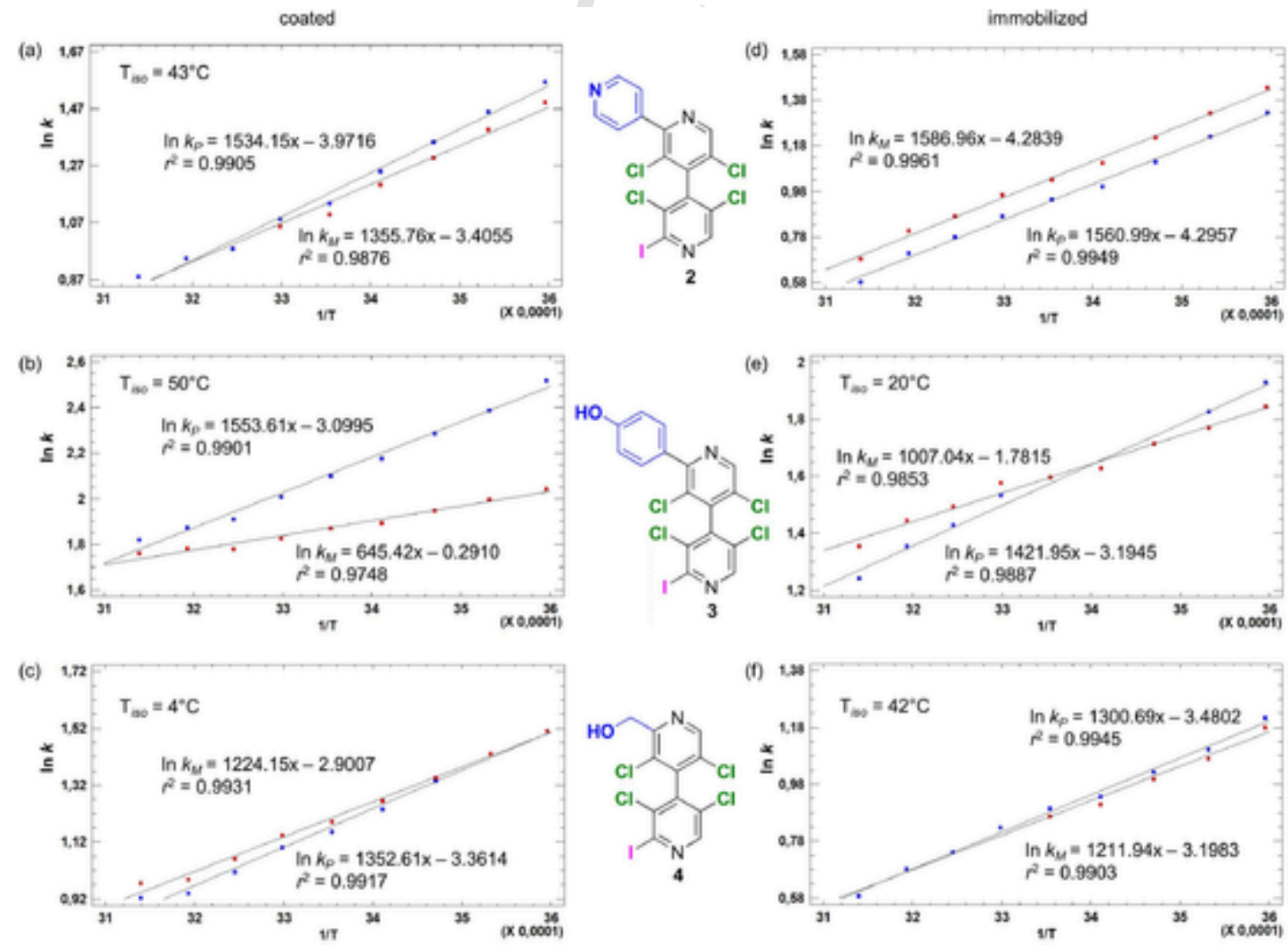

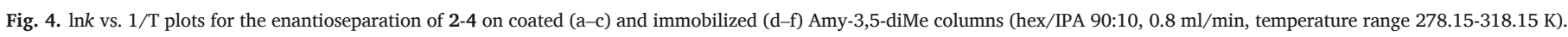


coated
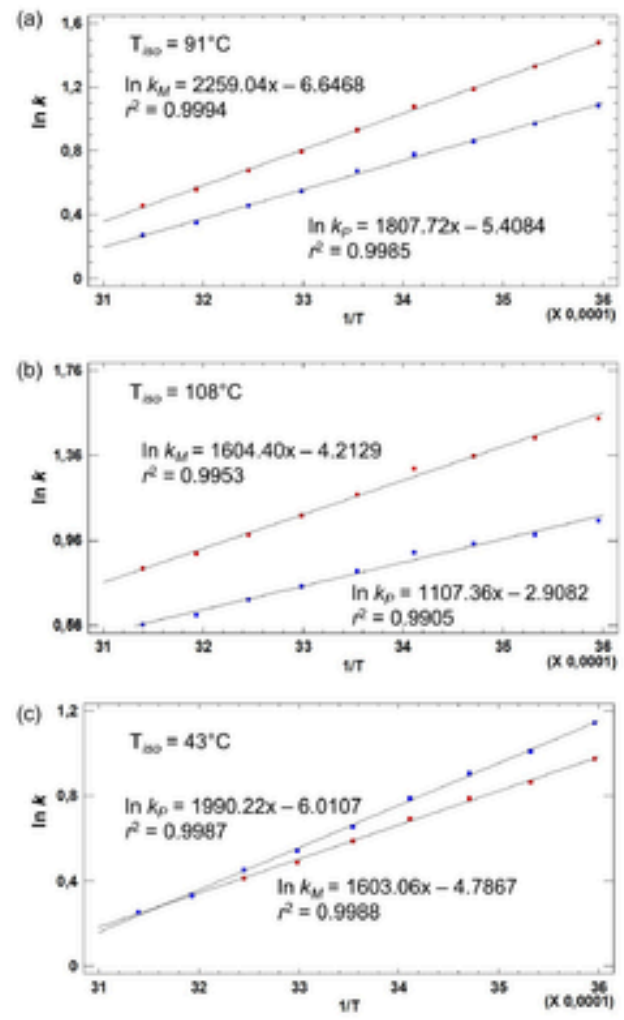

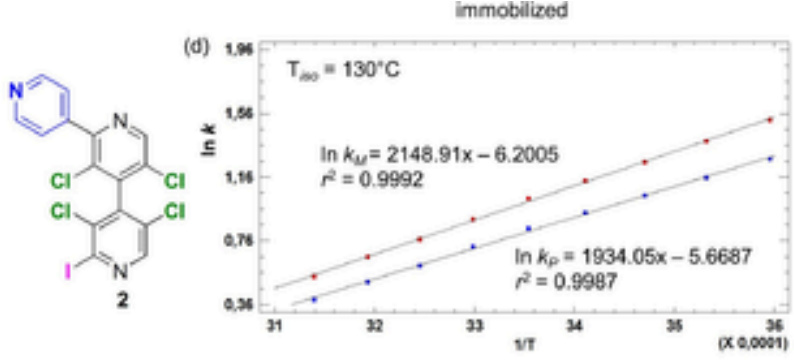

(e)
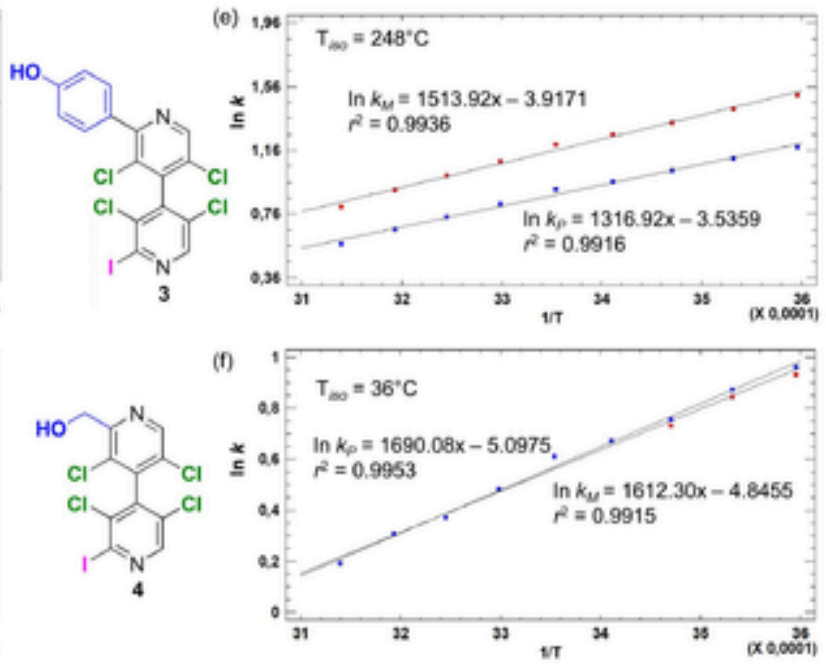

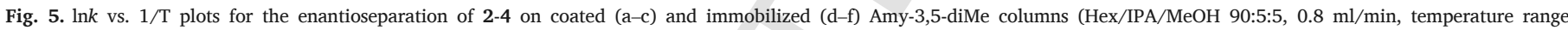
278.15-318.15 K).

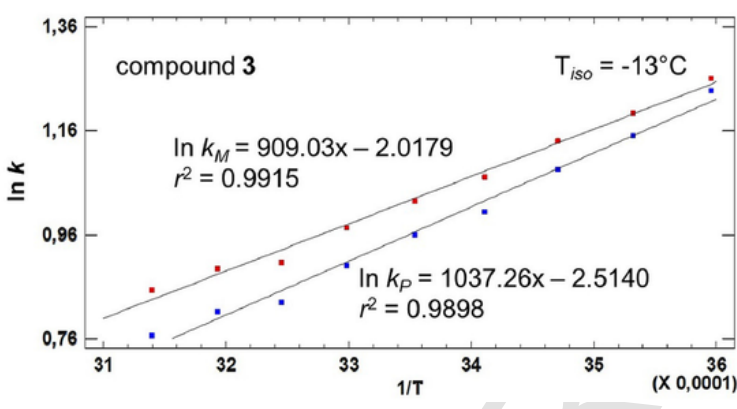

Fig. 6. $\ln k$ vs. $1 / \mathrm{T}$ plots for the enantioseparation of 3 on immobilized Amy-3-Cl-5Me column (hex/IPA 90:10, $0.8 \mathrm{ml} / \mathrm{min}$, temperature range 278.15-318.15 K).

i) compounds 2-4 showed different thermodynamic profiles on both columns, and the temperature-dependence pattern was observed to be a function of the 2'-substituent nature (Fig. 4).

ii) for compound 2 (2'- $\mathrm{R}=4$-pyridyl), enantioseparation was enthalpy-driven on both columns (Fig. 4a,d). Nevertheless, different thermodynamic profiles could justify the EEO reversal from $M-P$ to $P$ - $M$ observed on coated and immobilized columns. In the first case (Fig. 4a), a $\mathrm{T}_{I S O}$ of $43^{\circ} \mathrm{C}$ limits the enthalpic domain in the operative temperature range $5-45^{\circ} \mathrm{C}$, expecting an EEO reversal over $45^{\circ} \mathrm{C}$. On the immobilized column (Fig. 4d), the difference between the free energies associated with the adsorption of the enantiomers onto the CSP surface is essentially due to a negative enthalpy contribution, whereas the entropy term is positive and close to zero. Indeed, the enantioselectivity is almost independent of the temperature variation (Supplementary data, Fig. S2d).

iii) for compound 3 (2'- $\mathrm{R}=$ 4-hydroxyphenyl) different $\mathrm{T}_{\text {ISO }}$ values were calculated on the two Amy-3,5-diMe, being higher on the coated column $\left(50^{\circ} \mathrm{C}\right)$ (Fig. $\left.4 \mathrm{~b}\right)$ with respect to its immobilized ver- sion $\left(20^{\circ} \mathrm{C}\right)$ (Fig. 4e). On this basis, the enantioseparation was enthalpy-driven on the coated column, whereas two domains were observed on the immobilized column due to fact that the isoenantioselective temperature falls within the operative temperature range (Supplementary data, Fig. S3). For both columns, the observed EEO was $M-P$ in the enthalpic domains. For this thermodynamic scenario, the $\ln \alpha$ vs $1 /$ T showed the characteristic V shape (Supplementary data, Fig. S2e).

iv) compound 4 (2' $\mathrm{R}$ = hydroxymethyl) showed the same low enantioselectivity on the two columns $(\alpha=1.03)$, but reversal of EEO associated with two opposite thermodynamic profiles was observed (Fig. $4 \mathrm{c}$ and $\mathrm{f}$ ). Indeed, a $\mathrm{T}_{I S O}$ of $4^{\circ} \mathrm{C}$ was calculated on the coated column, which is lower than the temperature of isoelution observed in the immobilized Amy-3,5-diMe $\left(42^{\circ} \mathrm{C}\right)$. On this basis, in the operative temperature range $5-45^{\circ} \mathrm{C}$, the enantioseparation was entropy-driven $(P-M)$ on the coated column and enthalpy-driven $(M-P)$ on the immobilized one. In this regard, with the aim to verify the chromatographic behaviour over the limit of the entropy domain, the enantioseparation of compound 4 on the coated Amy-3,5-diMe was also performed at $0,-5$, and $-10^{\circ} \mathrm{C}$. Partial separation was observed at $-10^{\circ} \mathrm{C}$ with the expected reversal of EEO ( $M-P$, Supplementary data, Fig. S4).

With MeOH-containing MP, for compounds 2 (2'-R = 4-pyridyl) and 3 (2'- $\mathrm{R}=4$ 4-hydroxyphenyl) the operative temperature was below the computed $\mathrm{T}_{I S O}$ for all four enantioseparations (Fig. 5a,b,d,e), therefore the recognition process was enthalpy-driven on both coated and immobilized Amy-3,5-diMe columns (Supplementary data, Fig. S5). The introduction of $\mathrm{MeOH}$ as the organic modifier in the MP produced a relevant increase of $\mathrm{T}_{I S O}$ for both compounds. For compound 4 (Fig. $5 \mathrm{c}$ and $\mathrm{f}$ ), the introduction of $\mathrm{MeOH}$ in the MP increased the $\mathrm{T}_{I S O}$ from 4 to $43^{\circ} \mathrm{C}$ on the coated column thus increasing the limits of the enthalpic domain, whereas on the immobilized column an opposite effect 
with lower $\mathrm{T}_{I S O}$ variation from 42 to $36^{\circ} \mathrm{C}$ was observed. In the latter case, the lack of enantioseparation observed on the immobilized Amy-3,5-diMe at $25^{\circ} \mathrm{C}$ was due to the proximity of the operative temperature to the $\mathrm{T}_{I S O}$ calculated for 4 .

On the immobilized Amy-3-Cl-5-Me, only compound 3 was enantioseparated in the considered series. Interestingly, with this chlorinated selector, the calculated $\mathrm{T}_{I S O}$ was negative $\left(-13^{\circ} \mathrm{C}\right)$ (Supplementary data, Table S15) and the enantioseparation was thus entropy-driven in the range $5-45^{\circ} \mathrm{C}$ (Fig. 6).

By correlating the thermodynamic profiles with the structural features of analytes 1-4, the observed EEO reversals appeared dependent on the type of thermodynamic pattern, enthalpy- or entropy-driven, and two recognition mechanisms could be profiled for the enantioseparation of compounds 2-4 on the amylose-based columns: a) in the enthalpic domains, the EEO is $M-P$ for all compounds on all columns, likely due to HB-driven mechanisms; b) in the entropic domains, the EEO is always $P-M$ for all compounds, in this case a 'hydrophobic' mechanism driven by aryl rings and related $\pi-\pi$ interactions controls enantioseparation. In this regard, it is worth noting that $P-M$ was always observed as EEO for compound 1 which contains a phenyl group as distinctive 2'-substituent.

\section{Conclusions}

The results collected in this study show that 4,4'-bipyridines 1-4 can be successfully resolved within $20 \mathrm{~min}$ by using amylose-based chiral columns and Hex/IPA/MeOH 90:5:5 as MP (Supplementary data, Table S5).

In particular, the most challenging task concerned compound 4 (2'-R = hydroxymethyl), which could be baseline enantioseparated on the coated Amy-3,5-diMe by lowering the operative temperature to $5^{\circ} \mathrm{C}$. On the other hand, the baseline enantioseparations of compound 3 (2'-R = 4-hydroxyphenyl) on both coated and immobilized Amy-3,5-diMe could be performed within 15 minutes by raising temperature to 35 and $40^{\circ} \mathrm{C}$, respectively.

EEO reversals dependent on the nature of polar modifier, analyte structure, column-type, and temperature were observed for compounds 2-4. The comparison of the chromatographic behaviour of coated and immobilized Amy-3,5-diMe chiral columns under standard NP mode (Hex/IPA 90:10) confirmed that immobilization procedures induce subtle alterations in the selector generating a chiral platform with different recognition capability and thermodynamic profiles with respect to the coated selector. Interestingly, for compounds 2-4, the introduction of $\mathrm{MeOH}$ in the MP reduced the differences between the two types of selector, making their respective recognition mechanisms and thermodynamic profiles comparable. In particular, temperature-dependent enantioseparations of compound 3 performed in the range $5-45^{\circ} \mathrm{C}$ allowed us to identify for this compound thermodynamic profiles dependent on selector-type, CSP-type and MP.

Compounds 2-4 proved to be privileged structures to comparatively investigate the chromatographic behaviour of coated and immobilized amylose-based CSPs. Indeed, these compounds exhibited adaptability to different chromatographic systems, also generated by temperature-induced phase transitions of selector structure $[11,45]$. In our opinion, the key of this versatility is the simultaneous presence within the same molecule of different descriptors, namely HB sites, aryl groups, and hydrophobic regions able to promote different types of noncovalent contacts. On this basis, different mechanisms can be turn on or switch off depending on operative conditions and structural features of selector. In particular, the adaptable behaviour of compound 3 could be due to its favourable molecular properties, this compound showing the highest dipole moment, hydrophobic character, polarizability, isosurface area, and minor globularity (high ovality, $O$ ) with respect to $\mathbf{2}$ and $\mathbf{4}$ (Supplementary data, Table S4).

The possibility to modulate recognition ability of coated and immobilized polysaccharide-selectors by tuning temperature [11] paves the way to interesting scenarios for their utilization in enantiosepara- tion science. In this perspective, thermodynamic quantities estimated by van't Hoff analysis provide meaningful practical information in order to identify entropy-domains where increasing temperature can favour both selectivity and resolution.

\section{Declaration of Competing Interest}

The authors declare that they have no known competing financial interests or personal relationships that could have appeared to influence the work reported in this paper.

\section{Credit author contribution statement}

Paola Peluso: Conceptualization, Project administration, Data curation, Methodology, Writing - original draft, review \& editing; Barbara Sechi: HPLC analysis, Data curation; Giancarlo Lai: HPLC analysis, Data curation; Alessandro Dessì: DFT calculations; Roberto Dallocchio: DFT calculations; Sergio Cossu: Funding acquisition, Writing - review \& editing; Emmanuel Aubert: absolute configuration assignment, Writing - review \& editing, Robin Weiss: Syntheses; Patrick Pale: Syntheses; Victor Mamane: Syntheses, Funding acquisition, Writing - review \& editing; Bezhan Chankvetadze: Resources, Writing - review \& editing.

\section{Acknowledgement}

This work has been supported by Università Ca' Foscari Venezia, Italy (Dipartimento di Scienze Molecolari e Nanosistemi DSMN, ADIR funds). We thank the International Center Frontier Research in Chemistry (icFRC) and the Laboratory of Excellence for Complex System Chemistry (LabEx CSC) .

\section{Supplementary materials}

Supplementary material associated with this article can be found, in the online version, at doi:10.1016/j.chroma.2020.461303.

\section{References}

[1] B Chankvetadze, Polysaccharide-based chiral stationary phases for enantioseparations by high-performance liquid chromatography: an overview, in: G K E Scriba (Ed.), Chiral Separations: Methods and Protocols, Methods in Molecular Biology, 1985, Springer Science + Business Media, LLC, part of Springer Nature, 2019, pp. 93-126.

[2] J Shen, Y Okamoto, Efficient separation of enantiomers using stereoregular chiral polymers, Chem. Rev. 116 (2016) 1094-1138.

[3] E R Francotte, Polysaccharide derivatives as unique chiral selectors for enantioselective chromatography, Chimia 71 (2017) 430-450.

[4] M Lämmerhofer, Chiral recognition by enantioselective liquid chromatography: mechanisms and modern chiral stationary phases, J. Chromatogr. A 1217 (2010) 814-856.

[5] G K E Scriba, Chiral recognition in separation sciences. Part I: polysaccharide and cyclodextrin selectors, Trends Anal. Chem. 120 (2019) 115639.

[6] P Peluso, V Mamane, A Dessì, R Dallocchio, E Aubert, C Gatti, D Mangelings, S Cossu, Halogen bond in separation science: a critical analysis across experimental and theoretical results, J. Chromatogr. A 1616 (2020) 460788.

[7] M Maisuradze, G Sheklashvili, A Chokheli, I Matarashvili, T Gogatishvili, T Farkas, B Chankvetadze, Chromatographic and thermodynamic comparison of amylose tris(3-chloro-5-methylphenylcarbamate) coated or covalently immobilized on silica in high-performance liquid chromatographic separation of the enantiomers of select chiral weak acids, J. Chromatogr. A 1602 (2019) 228-236.

[8] T Zhang, P Franco, Analytical and preparative potential of immobilized polysaccharide-derived chiral stationary phases, in: G Subramanian (Ed.), Chiral Separation Techniques - a Practical Approach, third ed., Wiley-VCH, Weinheim, Germany, 2006, pp. 99-134.

[9] R Ferretti, A Mai, B Gallinella, L Zanitti, S Valente, R Cirilli, Application of 3 $\mu \mathrm{m}$ particle-based amylose-derived chiral stationary phases for the enantioseparation of potential histone deacetylase inhibitors, J. Chromatogr. A 1218 (2011) 8394-8398.

[10] N Beridze, E Tsutskiridze, N Takaishvili, T Farkas, B Chankvetadze, Comparative enantiomer-resolving ability of coated and covalently immobilized versions of two polysaccharide-based chiral selectors in high-performance liquid chromatography, Chromatographia 81 (2018) 611-621. 
[11] I Matarashvili, G Kobidze, A Chelidze, G Dolidze, N Beridze, G Jibuti, T Farkas, $B$ Chankvetadze, The effect of temperature on the separation of enantiomers with coated and covalently immobilized polysaccharide-based chiral stationary phases, J. Chromatogr. A 1599 (2019) 172-179.

[12] B Chankvetadze, Recent trends in preparation, investigation and application of polysaccharide-based chiral stationary phases for separation of enantiomers in high-performance liquid chromatography, Trends Anal. Chem. 122 (2020) 115709.

[13] A Felinger, Molecular dynamic theories in chromatography, J. Chromatogr. A 1184 (2008) 20-41.

[14] T Fornstedt, Characterization of adsorption processes in analytical liquid-solid chromatography, J. Chromatogr. A 1217 (2010) 792-812.

[15] Y B Band, Y Avishai, Density functional theory, Quantum Mechanics with Applications to Nanotechnology and Information Science, Ch. 15, first edition, Academic Press, Elsevier Ltd., Oxford, UK, 2013, pp. 871-889.

[16] A Dessì, P Peluso, R Dallocchio, R Weiss, G Andreotti, M Allocca, E Aubert, P Pale, V Mamane, S Cossu, Rational design, synthesis, characterization and evaluation of iodinated 4,4'-bipyridines as new transthyretin fibrillogenesis inhibitors, Molecules 25 (2020) 2213.

[17] P Peluso, V Mamane, E Aubert, S Cossu, Optimization of the HPLC enantioseparation of 3,3'-dibromo-5,5'-disubstituted-4,4'-bipyridines using immobilized polysaccharide-based chiral stationary phases, J. Sep. Sci. 36 (2013) 2993-3003.

[18] P Peluso, V Mamane, E Aubert, S Cossu, High-performance liquid chromatography enantioseparation of polyhalogenated 4,4'-bipyridines on polysaccharide-based chiral stationary phases under multimodal elution, J. Sep. Sci. 37 (2014) 2481-2489.

[19] P Peluso, V Mamane, E Aubert, S Cossu, Insights into the impact of shape and electronic properties on the enantioseparation of polyhalogenated 4,4'-bipyridines on polysaccharide-type selectors. Evidence of stereoselective halogen bonding interactions, J. Chromatogr. A 1345 (2014) 182-192.

[20] P Peluso, V Mamane, R Dallocchio, A Dessì, R Villano, D Sanna, E Aubert, P Pale, S Cossu, Polysaccharide-based chiral stationary phases as halogen bond acceptors: a novel strategy for detection of stereoselective $\sigma$-hole bonds in solution, J. Sep. Sci. 41 (2018) 1247-1256.

[21] H Koller, K-E Rimböck, A Mannschreck, High-pressure liquid chromatography on triacetylcellulose: characterization of a sorbent for the separation of enantiomers, J. Chromatogr. 282 (1983) 89-94.

[22] Y Shao, L F Molnar, Y Jung, J Kussmann, C Ochsenfeld, S T Brown, A T B Gilbert, L V Slipchenko, S V Levchenko, D P O'Neil, R A Di Stasio Jr., R C Lochan, T Wang, G J O Beran, N A Besley, J M Herbert, C Y Lin, T VanVoorhis, S H Chien, A Sodt, R P Steele, V A Rassolov, P E Maslen, P P Korambath, R D Adamson, B Austin, J Baker, E F C Byrd, H Dachsel, R J Doerksen, A Dreuw, B D Dunietz, A D Dutoi, T R Furlani, S R Gwaltney, A Heyden, S Hirata, C-P Hsu, G Kedziora, R Z Khalliulin, P Klunzinger, A M Lee, M S Lee, W Z Liang, I Lotan, N Nair, B Peters, E I Proynov, P A Pieniazek, Y M Rhee, J Ritchie, E Rosta, C D Sherrill, A C Simmonett, J E Subotnik, H L Woodcock III, W Zhang, A T Bell, A K Chakraborty, D M Chipman, F J Keil, A Warshel, W J Hehre, H F Schaefer, J Kong, A I Krylov, P M W Gill, M Head-Gordon, Advances in methods and algorithms in a modern quantum chemistry program package, Phys. Chem. Chem. Phys. 8 (2006) 3172-3191.

[23] M J Frisch, G W Trucks, H B Schlegel, G E Scuseria, M A Robb, J R Cheeseman, G Scalmani, V Barone, B Mennucci, G A Petersson, H Nakatsuji, M Caricato, X Hratchian, H P Li, A F Izmaylov, J Bloino, G Zheng, J L Sonnenberg, M Hada, M Ehara, K Toyota, R Fukuda, J Hasegawa, M Ishida, T Nakajima, Y Honda, O Kitao, H Nakai, T Vreven, J A Montgomery Jr., J E Peralta, F Ogliaro, M Bearpark, J J Heyd, E Brothers, K N Kudin, V N Staroverov, T Keith, R Kobayashi, J Normand, K Raghavachari, A Rendell, J C Burant, S S Iyengar, J Tomasi, M Cossi, N Rega, J M Millam, M Klene, et al., Gaussian 09, Revision B. 01, Inc.Gaussian, C.T. Wallingford, 2010.

[24] T Lu, F Chen, Multiwfn: a multifunctional wavefunction analyzer, J. Comp.Chem. 33 (2012) 580-592.

[25] T Lu, F Chen, Quantitative analysis of molecular surface based on improved marching tetrahedra algorithm, J. Mol. Graph. Model. 38 (2012) 314-323.

[26] P Peluso, V Mamane, E Aubert, A Dessì, R Dallocchio, A Dore, P Pale, S Cossu, Insights into halogen bond-driven enantioseparations, J. Chromatogr. A 1467 (2016) 228-238.

[27] P Peluso, V Mamane, E Aubert, S Cossu, High performance liquid chromatography enantioseparation of atropisomeric 4,4'-bipyridines on polysaccharide-type chiral stationary phases: impact of substituents and electronic properties, J. Chromatogr. A 1251 (2012) 91-100.

[28] B-H Kim, S U Lee, D C Moon, Chiral recognition of $N$-phthaloyl, $N$-tetrachlorophthaloyl, and $N$-naphthaloyl $\alpha$-amino acids and their esters on polysaccharide-derived chiral stationary phases, Chirality 24 (2012) 1037-1046.

[29] P Peluso, S Cossu, Comparative HPLC enantioseparation of thirty-six aromatic compounds on four columns of the Lux series: impact of substituents, shapes and electronic properties, Chirality 25 (2013) 709-718.

[30] Y Yamada, K Ohyama, G Onodera, M Kuriyama, N Kishikawa, N Kuroda, Molecular-shape selectivity by naphthalimido-modified silica stationary phases: Insight into the substituents effect of naphthalene on shape recognition and $\pi-\pi$ interactions via electrostatic potential, J. Chromatogr. A 1425 (2015) 173-179.

[31] G R Desiraju, P Shing Ho, L Kloo, A C Legon, R Marquardt, P Metrangolo, P Politzer, G Resnati, K Rissanen, Definition of the halogen bond (IUPAC recommendations 2013), Pure Appl. Chem. 85 (2013) 1711-1713.
[32] B Chankvetadze, Recent developments on polysaccharide-based chiral stationary phases for liquid-phase separation of enantiomers, J. Chromatogr. A 1269 (2012) 26-51.

[33] B Chankvetadze, L Chankvetadze, S Sidamonidze, E Kasashima, E Yashima, Y Okamoto, 3-Fluoro-, 3-chloro- and 3-bromo-5-methylphenylcarbamates of cellulose and amylose as chiral stationary phases for high performance liquid chromatographic enantioseparation, J. Chromatogr. A 787 (1997) 67-77.

[34] B Chankvetadze, C Yamamoto, Y Okamoto, Enantioseparation of selected chiral sulfoxides using polysaccharide-type chiral stationary phases and polar organic, polar aqueous-organic and normal-phase eluents, J. Chromatogr. A 922 (2001) 127-137.

[35] V Schurig, M Juza, Approach to the thermodynamics of enantiomer separation by gas chromatography. Enantioselectivity between the chiral inhalation anesthetics enflurane, isoflurane and desflurane and a diluted $\gamma$-cyclodextrin derivative, J. Chromatogr. A 757 (1997) 119-135.

[36] T Fornstedt, P Sajonz, G Guiochon, Thermodynamic study of an unusual chiral separation. Propranolol enantiomers on an immobilized cellulase, J. Am. Chem. Soc. 119 (1997) 1254-1264.

[37] G Guiochon, A Felinger, D G Shirazi, A M Katti, Fundamentals of Preparative and Nonlinear Chromatography, second ed., Academic Press, Boston, MA, 2006.

[38] J Samuelsson, R Arnell, T Fornstedt, Potential of adsorption isotherm measurements for closer elucidating of binding in chiral liquid chromatographic phase systems, J. Sep. Sci. 32 (2009) 1491-1506.

[39] L D Asnin, M V Stepanova, Van't Hoff analysis in chiral chromatography, J. Sep. Sci. 41 (2018) 1319-1337.

[40] A Sepsey, E Horváth, M Catani, A Felinger, The correctness of van't Hoff plots in chiral and achiral chromatography, J. Chromatogr. A 1611 (2020) 460594.

[41] T O'Brien, L Crocker, T Thompson, K Thompson, P H Toma, D A Conlon, B Feibish, C Moeder, G Bicker, N Grinberg, Mechanistic aspects of chiral discrimination on modified cellulose, Anal. Chem. 69 (1997) 1999-2007.

[42] C Panella, R Ferretti, A Casulli, R Cirilli, Temperature and eluent composition effects on enantiomer separation of carvedilol by high-performance liquid chromatography on immobilized amylose-based chiral stationary phases, J. Pharm. Anal. 9 (2019) 324-331.

[43] T Fornstedt, P Sajonz, G Guiochon, A closer study of chiral retention mechanisms, Chirality 10 (1998) 375-381.

[44] F Ianni, Z Pataj, H Gross, R Sardella, B Natalini, W Lindner, M Lämmerhofer, Direct enantioseparation of underivatized aliphatic 3-hydroxyalkanoic acids with a quinine-based zwitterionic chiral stationary phase, J. Chromatogr. A 1363 (2014) 101-108.

[45] F Wang, R M Wenslow Jr., T M Dowling, K T Mueller, I Santos, J M Wyvratt, Characterization of a thermally induced irreversible conformational transition of amylose tris(3,5-dimethylphenylcarbamate) chiral stationary phase in enantioseparation of dihydropyrimidinone acid by quasi-equilibrated liquid chromatography and solid-state NMR, Anal. Chem. 75 (2003) 5877-5885. 